\title{
Direct Detection of Exoplanets
}

\author{
J.-L. Beuzit \\ Laboratoire d'Astrophysique de Grenoble \\ D. Mouillet \\ Observatoire Midi-Pyrénées \\ B. R. Oppenheimer \\ American Museum of Natural History \\ J. D. Monnier \\ University of Michigan, Ann Arbor
}

\begin{abstract}
Direct detection of exoplanets from the ground is now within reach of existing astronomical instruments. Indeed, a few planet candidates have already been imaged and analyzed and the capability to detect (through imaging or interferometry) young, hot, Jupiter-mass planets exists. We present here an overview of what such detection methods can be expected to do in the near and far term. These methods will provide qualitatively new information about exoplanets, including spectroscopic data that will mature the study of exoplanets into a new field of comparative exoplanetary science. Spectroscopic study of exoplanet atmospheres promises to reveal aspects of atmospheric physics and chemistry as well as internal structure. Astrometric measurements will complete orbital element determinations partially known from the radial velocity surveys. We discuss the impact of these techniques, on three different time scales, corresponding to the currently available instruments, the new "Planet Finder" systems under development for 8 to 10 -m telescopes, foreseen to be in operation in 5 to 10 years, and the more ambitious but more distant projects at the horizon of 2020 .
\end{abstract}

\section{INTRODUCTION}

Since the discovery of a planet around a solar-type star ten years ago by Mayor and Queloz (1995), the study of exoplanets has developed into one of the primary research areas in astronomy today. More than 170 exoplanets have been found orbiting stars of spectral types $\mathrm{F}$ to $\mathrm{M}$, with a significant fraction in multi-planet systems (see the chapter by Udry et al. for a review). These exoplanets have been discovered using indirect detection methods, in which only the planet's influence on the host star is observed.

Indirect detection techniques include radial velocity measurements, which detect the movement of a star due to a planet's gravitational influence (see the chapter by Udry et al.; Marcy et al., 2003), photometric transit observations, which detect the variation in the integrated stellar flux due to a planetary companion passing through the line of sight to its host star (see the chapter by Charbonneau et $a l$.), as well as astrometry, which also detects stellar motion (Sozzetti, 2005), and gravitational microlensing, which involves unrepeatable observations biased toward planets with short orbital periods (Mao and Paczynski, 1991; Gould and Loeb, 1992).

Almost all of the currently known exoplanets have been detected by radial velocity measurements. Due to the unknown inclination angle of the orbit, only a lower limit of the mass can actually be derived for each individual planet candidate. The statistical distribution of exoplanets can still be obtained thanks to the large number of detections. These exoplanets typically have masses similar to those of the giant, gaseous planets in our own solar system. They are therefore generally referred to as Extrasolar Giant Planets, or EGPs. Since 2004, a few planets with minimum masses ranging between 6 and 25 Earth masses have been detected by radial velocity measurements (Butler et al., 2004; Rivera et al., 2005; Bonfils et al., 2005). Very recently, Beaulieu et al. (2006) have discovered a 5.5 Earth mass planet by gravitational lensing, assuming a mass of 0.2 Solar mass for the host star, based on the peak of the IMF at that mass.

These indirect methods have proven to be very successful in detecting exoplanets, but they only provide limited information about the planets themselves. For example, radial velocity detections allow derivation of a planet's orbital period and eccentricity as well as a lower limit to its mass due to the unknown inclination angle of the orbit. Photometric transit observations provide information about a planet's radius, and, with great effort, limited measurements of the composition of its upper atmosphere (Jha et al., 2000). In addition, large radial velocity surveys with sufficient precision only started about a decade ago. Thus, they are sensitive only to exoplanets with relatively small orbital periods, typically corresponding to objects at distance smaller 
than a few AUs from their parent stars. Currently, these surveys are completely insensitive to planets at separations comparable to those of Jupiter and Saturn in our solar system. Finally, the accuracy of radial velocity measurements strongly biases the detections based on the type of the host stars toward old, quite and solar mass (G-K) stars. Future extensive search for transiting planets will suffer similar biases regarding orbital periods and stellar types.

Direct detection and spectroscopy of the radiation from these exoplanets is necessary to determine their physical parameters, such as temperature, pressure, chemical composition, and atmospheric structure. These parameters are critically needed to constrain theories of planet formation and evolution. Furthermore direct detection enables the study of planets in systems like our own. In these respects, direct detection is complementary to the indirect methods, especially to the radial velocity technique.

However, direct observation of exoplanets is still at the edge of the current capabilities, able to reveal only the most favorable cases of very young and massive planets at large distances from the central star. (See Section 2.1). The major challenge for direct study of the vast and seemingly diverse population of exoplanets resides in the fact that most of the planets are believed to be $10^{6}$ to $10^{12}$ times fainter than their host stars, at separations in the sub-arcsecond regime. This requires both high contrast and spatial resolution.

Various instrumental approaches have been proposed for the direct detection of exoplanets, either from the ground or from space. In particular, several concepts of high-order adaptive optics systems dedicated to ground-based large (and small) telescopes have been published during the past ten years (Angel and Burrows, 1995; Dekany et al., 2000; Mouillet et al., 2002; Macintosh et al., 2002; Oppenheimer et al., 2003). Interferometric systems, using either a nulling or a differential phase approach have also been described (see Section 4). Space missions have been proposed, relying either on coronagraphic imaging (TPF-C observatory, see the chapter by Stapelfeldt et al.) or on interferometry (TPF-I and Darwin projects, e.g. Mennesson et al., 2005; Kaltenegger and Fridlund, 2005, and references therein).

In this chapter, we present the scientific objectives of the direct detection of exoplanets, concentrating on groundbased approaches. See the chapter by Stapelfeldt et al. for space-based approaches. We discuss the principle, performance and impact of the high contrast imaging and interferometric techniques on three different time scales, corresponding to the currently available instruments, the new "Planet Finder" systems under development and foreseen to be in operation in about 5 years, and the more ambitious projects on the 2020 horizon.

\section{SCIENCE}

\subsection{Direct detections}

Observations obtained in the past few years have reached contrasts compatible with the detection of Jupiter-mass planets in the most favorable case of very young ages $\left(10^{6}\right.$ to few $10^{7}$ years) when EGPs are still warm. Detection is typically possible outside of a $\sim 0.5$ " radius from the host star, corresponding to a few $10 \mathrm{AUs}$ at the typical distances of young nearby stellar associations, i.e. closer than $100 \mathrm{pc}$ (Zuckerman and Song, 2004).

A few adaptive optics surveys of some of these associations conducted in recent years (e.g. Chauvin et al., 2002; Neuhäuser et al., 2003; Brandeker et al., 2003; Beuzit et al., 2004; Masciadri et al., 2005) indeed resulted in the first three direct detections of exoplanet candidates. These discoveries include a companion around the brown dwarf 2MASSWJ 1207334-393254 (hereafter 2M1207), a member of the young TW Hydrae Association, by Chauvin et al. (2005a), around the classical TTauri star GQ Lup by Neuhäuser et al. (2005) and around AB Pic, a member of the large Tucana-Horologium association by Chauvin et al. (2005b). Fig. 1 illustrates the detection of two of these planet candidates, 2M1207 B and GQ Lup.

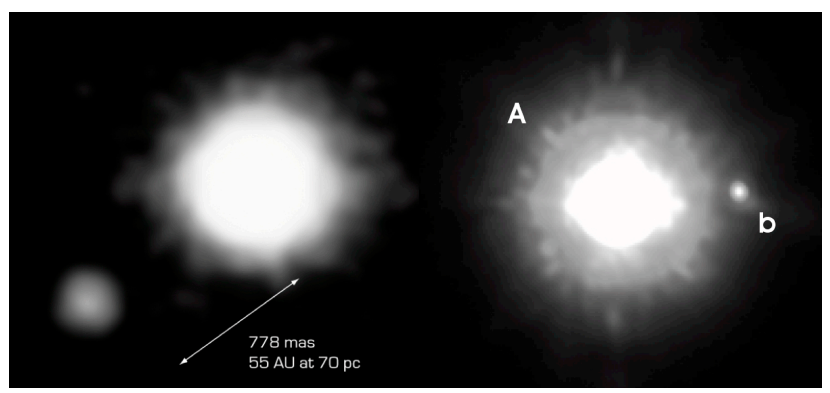

Fig. 1. - Examples of detection of planetary mass objects using current AO instruments. Left: 2M1207 found by Chauvin et al. (2005a). Right: GQ Lupi found by Neuhäuser et al. (2005). Both objects are estimated by some researchers to have planetary mass. See Section 2.1 for more detail.

The analyses of the proper motions, colors and low resolution spectra of these objects definitely confirm their status as co-moving, very low mass, companions. An estimation of the actual mass of these companions requires the determination of their luminosity and temperature through theoretical models, such as the Tucson (Burrows et al., 1997) and Lyon (Chabrier et al., 2000; Baraffe et al., 2002) models. These models are known to be uncertain at early ages, typically $<100$ Myrs, and the derived mass estimates, and therefore whether these companions are brown dwarfs or planets should be considered with caution (see Neuhäuser, 2006).

In any case, these observational results can now be directly compared to theoretical models. Such detections at very large orbital separations (55 to $250 \mathrm{AU}$ ) also possibly constrain the formation mechanisms of such planetary mass objects. See, for instance, the chapters by Lissauer et al., Durisen et al., Papaloizou et al. and Chabrier et al. for a discussion of these mechanisms.

These first direct detections are very encouraging and they clearly open a new window for exoplanet characterization. As with the radial velocity techniques, meaningful astrophysical results can only be derived when a larger sam- 
ple of observations will be available. The rest of this paper presents a wider perspective of the primary goals of such an approach, the expected science impact, and the corresponding observational requirements.

\subsection{Primary Science Goals}

In order to test these theories, it is crucial to obtain both statistical and direct information on these planets. A variety of observational techniques, direct and indirect, must ideally be used in parallel to reach this goal. As mentioned above, indirect detection methods suffer from ambiguities in interpretation: for instance, the observation of an occultation (or transit) provides the radius of the companion but without knowing the object's mass, it is impossible to know, in many cases, whether the object is in fact a planet, a brown dwarf (whose radii are similar to EGPs) or white dwarf (whose radii are similar to Earth-sized planets). Furthermore, radial velocity techniques are limited to only certain types of stars. Direct detection, in contrast, can survey, in theory, any type of star, including searches around degenerates such as white dwarfs and brown dwarfs for planetary mass companions. Direct detection is also far more efficient, even for long period planets, since it requires only two or three observations to confirm a detection, and only one to achieve spectra, rather than the multitude of observations over long periods of time necessary for radial velocity searches.

Direct detection of exoplanets in various planetary systems represents a great leap in our knowledge of these objects, as well as a rigorous maturation of the field. Some of the expected scientific returns from these observations are detailed below.

1. A removal of the $\sin i$ ambiguity that plagues the radial velocity measurements will not only remove statistical biases inherent in this technique (e.g. Han et al., 2001), but it also tests whether planetary systems generally have a single orbital plane for all planets (as seen in our solar system). This is a crucial aspect of formation theories (e.g. Butler et al., 1999).

2. Detection of planets around active stars is only possible through direct detection, and perhaps marginally by astrometry and transit monitoring. In particular young stars, which are choice targets for the study of planet formation, are particularly active. These kinds of studies can lead to an understanding of planet evolution.

3. Complete orbit determination leads to improved statistical understanding of planets as a function of mass, parent star type and age (i.e. orbital evolution). A significant fraction of the orbit must be covered for such studies, requiring long term monitoring campaigns commensurate with that of indirect methods (10 to 20 years).
4. Measurement of planet colors will constitute the first direct tests of atmospheric models, and will provide information on the presence of clouds in the planetary atmosphere, for instance.

5. Determination of the intrinsic luminosity of planets at various ages will yield additional constraints on models of planet evolution.

6. Spectroscopy of exoplanets. Surprisingly, the heavy element composition of solar gas giants is still poorly understood (e.g. Guillot, 1999). Spectral features of $\mathrm{CH}_{4}, \mathrm{NH}_{3}$ and $\mathrm{H}_{2} \mathrm{O}$ are observed at resolutions $\mathrm{R} \sim 20-30$. For brown dwarfs and luminous planets, narrow features of $\mathrm{KI}, \mathrm{NaI}$ and $\mathrm{FeH}$ are observed in the J-band and require $\mathrm{R} \sim 1000$ to be characterized. Major changes in spectral appearance occur as the amount and height of dust in the atmosphere changes, significantly modifying the heavy elements available for formation of molecules (Burrows and Sharp, 1999). Of particular interest is the comparison of these features in different planets along with such information as the metallicity of the parent star.

7. Measurement of spectral variability in planets due to activity or weather. Planets with effective temperatures between 200 and $300 \mathrm{~K}$ are expected to have atmospheres dominated by water clouds (e.g. the Earth and Guillot, 1999). Such clouds yield activity detectable by high dynamic range observations through three interrelated processes: $(i)$ inhomogeneities and rotation, (ii) meteorological activity, and (iii) the presence of dynamical waves. The third process may be the most promising, as it is thought to be responsible for the presence of Jupiter's fivemicron hot spots. In the case of planets with water clouds and a higher effective temperature, the effect should be more pronounced and displaced to shorter wavelengths.

8. Detection of exoplanetary ring systems. From our current limited statistics (i.e. the solar system), a substantial fraction of the discovered gas giants may have rings. These can be detected with polarimetry. In addition, changes in the projection of the rings with orbital phase will produce changes in a planet's spectrum. The effect of rings on planets where reflected light is an insignificant fraction of the emergent radiation must still be understood theoretically (i.e. how does luminosity modulate or degree of polarization change), but for systems dominated by reflected star light, the impact of rings has been evaluated (Figs. 3 to 16 in Arnold and Schneider, 2004). Luminosity modulation and Polarization depend on ring geometry and opacity. In some configurations these effects may augment detectability of the planet.

9. Polarimetry of planets in general. Although the fractional polarization from self-luminous planets is ex- 
pected to be very small (typically lower than 1\%), significant asymmetries in cloud distributions can induce higher polarization. In reflection, more than twenty percent polarization is seen at some orbital phases in the solar system. For a planet at the very short orbital distance of $0.3 \mathrm{AU}(0.1$ " at $3 \mathrm{pc})$, with $20 \%$ polarization and an albedo similar to that of Jupiter (0.3), the star-planet contrast will be of the order of 17.6 magnitudes, with a strong dependence on the orbital phase and system inclination. Such systems might be the first exoplanets imaged in reflected light.

10. Detection of moons. If our solar system is any indication of planets in general, most exoplanets will harbor moons. These can be detected in the future though searches for transits of the systems found, particularly in edge on configurations. Moons the size of Earth and even smaller may be detectable with $1 \%$ photometry of EGPs.

11. Evidence for biological activity. It would be irresponsible for us not to mention the possibility that some of the first signs of life outside the solar system may come in the distant future from spectroscopic observations of planets. A cottage industry of theorists modeling the chemical disequilibrium induced by biological activity on planets has emerged in anticipation of such results, and long term monitoring of the Earth-shine spectrum is designed to build up a library of spectra (at least of a 4.6 Gyr Earth-like planet) with which to compare future exoplanet spectra (e.g. Kasting and Catling, 2003; Woolf et al., 2002).

These science objectives indicate an incredibly wealthy field, one that routinely draws unprecedented interest from the public and is responsible for many new students entering astronomy. The record attendance at the conference at which this paper was presented is the best testimonial to this statement. The bottom line is that this field of research is broad, interdisciplinary and exciting in that it is attacking some of the most fundamental questions science can ask. A galaxy of worlds awaits our inspection. The future of astrophysics and planetary science lies in comparative exoplanetary science.

\subsection{Survey Design}

The frequency of planets in the mass and period ranges accessible to the proposed techniques is essentially unprobed (Fig. 2). This means that understanding planets in general requires collection of a large sample, through which salient properties and generalizations can be made and tied to theory. Direct observations require extensive surveys of hundreds of target stars. Such dedicated surveys must be optimized to ensure observing efficiency and data consistency compatible with statistically relevant analyses. Indeed advanced survey design and optimization is already being explored, in particular by Brown (2005) in the context of the Terrestrial Planet Finder. However, some simpler conceptions of surveys can be imagined, depending on the type of observation, expected system performance and telescope design. The following is an expurgated, but representative, list of such surveys.

1. Young and nearby stars: young nearby stellar associations are ideal targets for the direct detection of exoplanets, since $(i)$ they are typically closer than 100 pc and therefore allow the exploration of their circumstellar environment at very small distances from the host star, (ii) sub-stellar objects are hotter and brighter when young ( $\sim 10 \mathrm{Myr})$ and can be more easily detected than evolved companions, because the contrast between star and planet is smaller. The first direct detection of planetary mass objects used such associations to induce an age bias in the sample (see Section 2.1 above).

2. Nearest active stars: moderately young stars (younger than $\sim 1 \mathrm{Gyr}$ ) in the solar neighborhood (within $50 \mathrm{pc}$ of the Sun) offer major advantages for direct imaging due to their proximity and favorable contrast (again due to youth). For the most active of these stars, radial velocity detection of planets is very difficult if not impossible since the stars exhibit large intrinsic rotational velocities. Direct detection is, thus, the only possibility for exploring the planetary systems of these stars. For stars with distances from 10 to $50 \mathrm{pc}$, translating into separations of 1 to $100 \mathrm{AU}$ or periods of 1 to 1000 years for a solar mass primary, direct observation probes a separation range much larger than, and complementary to, that of radial velocities. For stars closer than $10 \mathrm{pc}$, radial velocity and direct imaging techniques overlap, allowing precise mass and luminosity estimates. These feed directly into an observed mass-luminosity relation. Finally, the closest of these stars will also offer the only opportunities for detecting planets by reflected light at short orbital distances in the near future.

3. Late-type stars (M dwarfs): these stars represent even more favorable targets for direct detection since the contrast between the star and the planet is much lower.

4. Stars with planets known from radial velocity surveys, especially stars that exhibit long term residuals in their radial velocity curves: such residuals indicate the presence of a more distant companion, perhaps more easily found through direct methods. When direct imaging instruments come into operation, we expect that the sample of planets discovered by radial velocity techniques will have increased significantly and include planets with longer periods. Such planets are at larger separations from their stars, which is favorable for direct detection. 


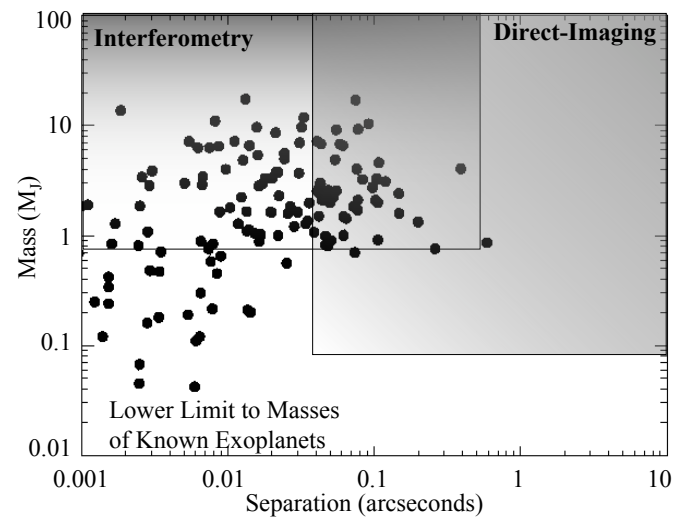

Fig. 2.- Mass versus separation diagram illustrating the complementarity of the different techniques used for extra-solar planet detection. The black circles indicate known planets discovered through indirect methods. The shaded regions show the approximate parameter space accessible, by 2020, to the direct techniques employing interferometry and high resolution imaging. Both direct and indirect techniques are complementary. See text for a more complete discussion.

\subsection{Key Scientific Requirements}

The scientific requirements driving the design of instruments for the direct exoplanet detection are based on the size of our own solar system (which determines the angular resolution and field of view needed to survey the nearest 1000 or so stars), the spectra of planets in our solar system, and theoretical models of EGPs (e.g. Burrows, 2005, and references therein, which set contrast requirements).

1. Sensitivity to wavelengths from 0.6 to $2.5 \mu \mathrm{m}$ : Giant gaseous planets are dominated by $\mathrm{CH}_{4}$ features in the $\mathrm{J}$ and $\mathrm{H}$ bands, while for terrestrial planets, the wavelength range 700 to $900 \mathrm{~nm}$ is particularly interesting, especially with astrophysically unique features like the $\mathrm{O}_{2}$ band. Wavelengths shorter than $700 \mathrm{~nm}$ have enhanced polarization in solar system objects, feeding into some of the science objectives mentioned above. Observations longwards of $2.5 \mu \mathrm{m}$ from the ground are heavily impeded by the terrestrial atmosphere, and are restricted to space-based observations.

2. Extremely high contrast: Young EGPs are believed to be typically 15 magnitudes $\left(10^{6} \times\right)$ fainter than their host stars, while an analog of the Earth is between 25 and 35 magnitudes $\left(10^{10}\right.$ to $\left.10^{14} \times\right)$ fainter, depending on the wavelength of observation (e.g. Segura et al., 2005).

3. Very high angular resolution: Access to angular separations as small as 20 mas is needed to resolve the radial scale of the Earth's orbit at a distance of $25 \mathrm{pc}$, within which there are thousands of stars to survey. This angle is also called the "inner working angle."

4. Total field-of-view extending to 2" to 4" in diameter: A field-of-view of 2" corresponds to an exploration region of $1 \mathrm{AU}$ in radius at $1 \mathrm{pc}$, roughly the distance to the nearest star. A field-of-view of 4" covers a region similar to the scale of our own solar system at distances of around $10 \mathrm{pc}$.

5. Relative astrometry at better than 10 mas precision: This permits discrimination of background objects from bona fide companions and accurate measurement of orbital motion on time scales of less than a few years around stars within $10 \mathrm{pc}$.

Early instruments in this endeavor will be limited in many respects. Achieving these goals requires an acute awareness of the technology available. For example, the sensitivity of high resolution adaptive optics approaches (which will be discussed in more detail below) is limited to stars with visual magnitudes of about 9 or 10 , and interferometers struggle to operate effectively on stars 4 or 5 times as bright. Clearly, extending this performance to fainter stars is necessary to enable surveys of thousands of stars. In addition, due to the faintness of the planets and the various noise sources impeding their detection, spectral resolution over the desired wavelength range will be limited to $\mathrm{R} \sim 30$ typically in the near term. Differential polarization from the ground is already making progress with the detection of protoplanetary disks and is planned to be included in the upcoming ground-based exoplanet imaging systems. Ultimately, much of this work will have to be done in space. However, space based missions have other limitations, such as duration, which means that the ground-based observations will always play a complementary role. The space based missions are described in the chapter by Stapelfeldt et al.

\section{HIGH CONTRAST IMAGING}

\subsection{System Overview}

The primary challenge in imaging exoplanets is the presence of a star at the center of the field of view and up to $10^{12}$ times brighter than the planet. Eliminating the light of this star without damaging that of the planet represents a major technical challenge and, in the case of imaging (interferometry is discussed in Section 4), requires new technology. In particular we identify the following challenges:

1. Correct the point spread function (PSF): (i) to confine the disturbing stellar light into a coherent, diffraction-limited pattern that is understood and controlled and can then be canceled (see next item), (ii) to concentrate the planetary flux, making the planet's peak brightness higher relative to the background 
due to the star light, and (iii) to reduce the incoherent stellar halo. If $S$ is the image Strehl ratio (which is essentially the fraction of coherent energy in this regime), then the ratio of planet brightness to stellar halo background scales as $S /(1-S)$. Note that with this asymptotic function, very large gains are made as $S \rightarrow 1$.

2. Cancel the coherent part of the stellar PSF by coronagraphy: This reduces the fundamental Poisson noise limit and dramatically relaxes constraints on PSF calibration, such as flat-field accuracy and stability.

3. Calibrate the residual (usually incoherent) stellar halo including residuals from atmospheric effects (evolving on ms time scales) but also much slower instrumental effects. Any a priori information able to distinguish an artifact from a planetary signal is to be used in this case for calibration purposes, such as the point-like shape of the planet, spectral and/or polarization signatures, or, perhaps the difference in coherence properties between the stellar halo and planet wave fronts.

Actually, these three steps are closely entangled in any practical system design: AO correction for PSF improvement contributes to measurements and calibration of the PSF halo. Such information can be used to improve stellar halo suppression in real time, for example. In any case, these three steps are the fundamental guiding principles in instrument design and data analysis.

\subsection{Current State of the Art: Necessary Subsystems}

3.2.1 Correction: Adaptive Optics. The primary source of disturbances to PSF quality is due to wave front perturbations imposed during propagation through the inhomogeneous atmosphere. AO is a technique that corrects these disturbances. The perturbations are measured with a dedicated wave front sensor and corrections are applied by an optically conjugated deformable mirror (DM) in real-time. The maximum performance for a given system, usually quantified as $S$ (above), is limited by technological constraints such as the number of actuators of the DM, the servo loop bandpass, and the overall system stability and calibration accuracy. The whole system acts as a filter, correcting most low temporal $(>10 \mathrm{~ms})$ and spatial frequencies $(>20 \mathrm{~cm})$ of the wave front perturbations. Remaining high spatial frequency defects translate in the image plane as a residual seeing-like halo extending outwards from the "control radius" $\lambda / 2 d$ (where $d$ is the inter-actuator spacing as projected on the telescope primary; Oppenheimer et al., 2003). Within this AO "control radius," the departure from a pure diffraction pattern is due to remaining very fast wave front variations and high frequency aliasing.

The typical performance achieved by current systems is of the order of $S \sim 50-60 \%$ on 8-m telescopes (Clénet et al.,
2004; van Dam et al., 2004; Stoesz et al., 2004) in the nearinfrared and up to $90 \%$ on 4-m telescopes (Oppenheimer et al., 2004). $S \sim 93 \%$ in the near-infrared on 8-m telescopes is the operating goal of the next generation of instruments, requiring technological improvements. Such improvements include wave front sensor detectors, micro deformable mirrors, and a new generation of real-time calculator, as a few examples.

With a reasonable inter-actuator spacing corresponding to $d \sim 10-20 \mathrm{~cm}$ (matched to the approximate Fried parameter for the telescope and site), the corrected region of the image extends to 0.8 " radius from the central star, which is well suited for planet searches (Section 2.4). Progress on wave front sensor detectors allows achieving correction on stars as faint as 11th mag., depending on the wavelength used for guiding, even with the required spatial and temporal sampling. This is compatible with investigations around a large target sample as discussed in Section 2.

When trying to reach even higher levels of correction $(S>90 \%)$, new limiting factors appear and must be taken into account. This includes atmospheric scintillation effects, and wave front perturbation chromatism, to name only a few. See for instance Dekany (2004) or Fusco et al. (2005) for in-depth discussions of these effects.

3.2.2 Cancellation: Coronagraphy. The basic purpose of coronagraphy (Lyot, 1939; Sivaramakrishnan et al., 2001) is to cancel the coherent part of the stellar energy over the observed field of view. In addition to the intrinsic merit of reducing the Poisson noise in the PSF wings (by decreasing the number of photons in the image), coronagraphy also reduces the interference between the residual incoherent and coherent wave fronts that form pinned speckles (Bloemhof et al., 2001; Aime and Soummer, 2004). This strongly relaxes the constraints on the quality of all optical components located after the coronagraphic devices.

Considering a well-corrected incoming wave front, when the phase defects remain small, the corresponding image can be approximated by the sum of a pure diffraction pattern (Airy pattern in the case of a circular aperture) and of an incoherent halo (shaped as the phase defect power spectrum density). In a perfect coronagraph the diffraction pattern is completely removed. In practice, however, no perfect coronagraph can be built and trade-offs must be made.

The important parameters for a coronagraph are $(i)$ the level of star light rejection, (ii) the inner working angle, and (iii) the transmission of the off-axis planet light.

Various coronagraphic concepts have been proposed in recent years, supported by impressive efforts in simulation and prototyping. These concepts include amplitude focal masks coupled with pupil stops or amplitude pupil masks, phase masks, pupil amplitude separation and recombination (e.g. Soummer et al., 2003; Soummer, 2005; Guyon et al., 2005; Baudoz et al., 2005). These concepts present various levels of efficiency and sensitivity with respect to chromatism, pupil shape, residual image defects and residual tilt.

Laboratory experiments have convincingly demon- 
strated that, for future systems, the limitation should essentially come from the residual halo associated with phase defects rather than from the efficiency of the coronagraph itself (Trauger et al., 2004). This preliminary conclusion should be revisited for even more ambitious projects (whether based on the ground or in space).

3.2.3 Calibration: Differential Techniques. Several differential techniques have been proposed combining adaptive optics systems and coronagraphs, including differential imaging instruments (Lenzen et al., 2004; Close et al., 2005 for the Simultaneous Differential Imager, or SDI, on NACO), Integral Field Spectrographs, or IFS (Berton et al., 2006), and differential polarimeters (Gisler et al., 2004).

In the case of differential detection, the final performance is not limited by remaining wave front phase defects resulting in speckles that could mimic a faint planet, because these techniques are designed to remove such speckles. Instead the limitations are due to the much smaller noise induced by the differences in the various measurement channels (see also differential phase measurement in interferometry which uses a similar approach, in Section 4).

In spectral differential techniques, these limitations come in particular from (i) overall chromatic effects (speckle structure chromatism, residuals of the atmospheric dispersion correction, potential coronagraph chromatism, impact of the optical beam-shift due to atmospheric refraction, and defects in optical components located before the dispersion corrector, and (ii) distinct optical aberrations if distinct optical paths are involved (such as in SDI). When looking for very high contrasts, the remaining level of differential aberrations is a critical issue. In particular, it should be noted that these differential aberrations add to common aberrations: the impact on the image is then a combination of both types of aberrations and scales as $\sigma_{\text {common }}^{2} \times \sigma_{\text {differential }}$. As already underlined in the previous paragraphs, this strongly reinforces the requirement for very high image quality on the coronagraph.

The information obtained with spectral differential techniques is particularly well suited to the search and study of cold giant planets exhibiting in particular deep and low resolution molecular absorption features in the near-infrared (e.g. $\mathrm{CH}_{4}$, Oppenheimer et al., 1995, 1998). But, on the other hand, these techniques are likely to become much less efficient when searching for terrestrial type exoplanets in the future (especially when observing from the ground where the atmospheric features of the exoplanet are likely to significantly overlap with our own atmosphere absorption).

Differential polarimetry permits a similar approach, when comparing simultaneous images in two distinct polarization states. Difficulties associated with the atmospheric dispersion disappear here, but similar limitations related to differential instrumental polarization are encountered in this case if the images in the two polarization states are separated and propagated through distinct optical components. Such limitations were already encountered in existing in- struments for very high polarization accuracy (such as in solar physics). A solution has been proposed in the ZIMPOL concept (Gisler et al., 2004) with the use of an high frequency ( $\mathrm{kHz}$ regime) polarization switch on a single optical path. In that case, a polarization accuracy of $10^{-6}$ could be reached, that can translate into star-to-planet contrasts higher than $10^{-8}$. For such a performance level, the fundamental photon noise limit dominates with the consequence that only the brightest nearby stars (until the advent of the 30-m class Extremely Large Telescopes), can be surveyed. Also, the most favorable potential planetary targets would be close-in $(<1 \mathrm{AU})$ planets with a significant reflected (and polarized) light component.

In principle any defect measured and identified as being an instrumental artifact by differential measurements, could be corrected by a high accuracy corrective device in a closed loop. In that sense, such a technique is very similar to the two-stage adaptive correction systems being studied now. For example, such dual-stage systems are under consideration in the Planet Finder projects just beginning (Macintosh et al., 2004 for the Gemini system and Beuzit et al., 2006 for the VLT project).

\subsection{Achieved and Expected Performance}

3.3.1 Existing Systems. Today, the 4-m and 8-m class telescopes are equipped with instruments providing diffractionlimited imaging in the near-infrared. Even if they remain "general-use" instruments, deep investigations of the outer (typically $>0.5$ ") stellar environments can be carried out with classical coronagraphy and/or saturated images, opening the observational window to direct detection of orbiting planets. Accordingly, these searches must primarily focus on distant massive planets around young and low-mass stars, with extensive surveys of the newly identified young nearby stellar associations. See Section 2.1 for a discussion of the first direct detections obtained with the NACO instrument on the VLT.

Fig. 3 shows an example of an image from the Lyot Project, a dedicated system comprising an optimized Lyotstyle coronagraph behind a high-order AO system on a 3.6$\mathrm{m}$ telescope (Oppenheimer et al., 2004). This project is currently surveying nearby stars with contrast ratios in the H-band up to $10^{-6}$ within 2" of the central star and has implemented a differential polarimeter with a sensitivity to polarized objects up to 15 times fainter. Within two years an IFS will be installed behind this system to provide early results on much fainter unpolarized companions.

The definite scientific impact of the current search programs, on a very short time scale, provides only a glimpse into the existing EGPs in the outer environments ( $>$ few 10 AUs) of certain stellar classes. Even if the expected number of detections of planetary mass objects will not allow refined statistics, first indications on the existence and separation of such objects will be extremely valuable. For positive detections, comparison with models (including both internal structure and atmospheres of cool objects) will ad- 


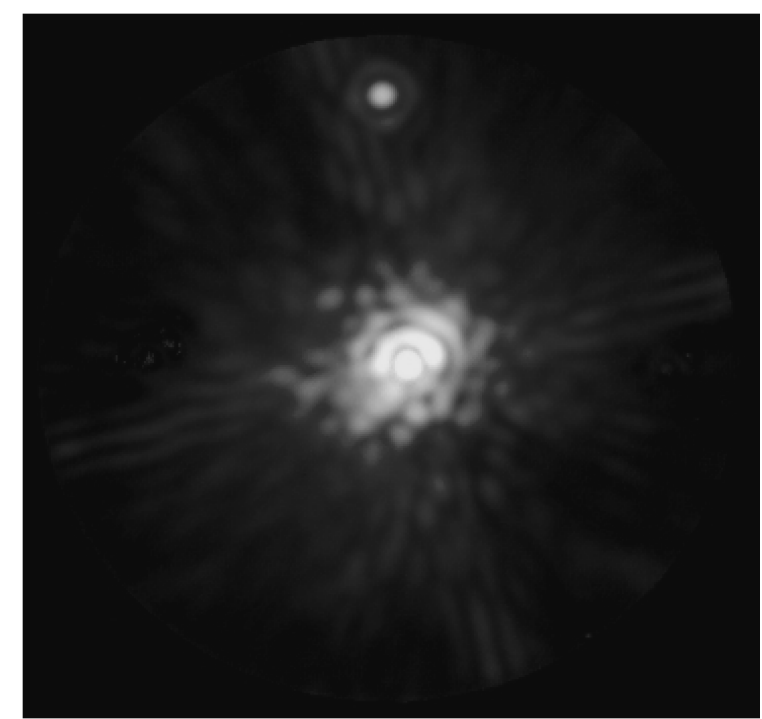

Fig. 3.- An H-band image from the Lyot Project Coronagraph, an optimized, diffraction-limited, classical coronagraph fitted behind an AO system with $d=10 \mathrm{~cm}$ on a $3.67-\mathrm{m}$ telescope, currently the highest order AO correction available (Oppenheimer et al., 2004). The companion (confirmed through common proper motion astrometry) to this nearby star is 11.3 mag. $\left(10^{-4.5}\right)$ times fainter than the star at a separation of 1.8 ". Greater contrast with this system is possible with differential polarimetry.

dress the questions of temperature, composition (molecules and dust) and evolution through near-infrared colors, low resolution spectroscopy, and estimations of bolometric luminosities. However, to gain access to a larger sample of targets and to smaller separations, in the 1 to $10 \mathrm{AU}$ domain, future instruments designed for much high contrast and smaller inner working angles are required.

3.3.2 Instruments Under Development. As mentioned above, optimized high contrast instruments require a consistent and well focused global design. Far superior performance is expected on existing telescopes using dedicated instruments instead of the "general-use" AO systems. Such dedicated planet hunting instruments are currently under development, targeted for the study of the inner environment of bright objects: such as NICI (Ftaclas et al., 2003), Subaru's Hi-CIAO, Gemini's Planet Imager (Macintosh et al., 2004), and VLT's Planet Finder (Beuzit et al., 2006; Fig. 4). These instruments will gradually become operational with increasing performance between 2006 and 2010.

With performance of $10^{-6}$ to $10^{-7}$ in contrast at 0.5 " from the star or closer, Jupiter mass companions will be accessible around a large number of young $(<100 \mathrm{Myr})$ stars. Also, slightly more massive planets $\left(5-10 M_{J}\right)$ could be detected around older and closer stars at shorter physical separations, in the 1 to $10 \mathrm{AU}$ range. The increased sensitivity of the new generation of wave front sensor detectors permit AO correction for host stars with magnitudes up to 12 with far better precision. This allows surveys to cover several hundred stars.
This generation of instruments, mostly operated in survey mode, rather than general standard small allocations of time for pointed observations, will definitely improve the statistical understanding of the population of EGPs, covering a far broader range of orbital separations range and stellar types. These studies are in direct complement to previous RV and photometric transit searches. Because of the improved statistics, it will be possible to test the impact of parameters such as the stellar age, mass, metallicity, as well as the presence of other (inner or outer) planets on the planet population and to place the brown dwarf and planet in relative scientific context.

Long integration times on the detected planets will provide a finer characterization of their spectra at low spectral resolution $(R \sim 30)$. For the closest separations $(\sim 1-5$ $\mathrm{AU})$, monitoring the orbital motion permits determination of refined orbital parameters, variability and derivation of dynamical masses.

Finally, direct detections of the reflected light of close-in $(<1 A U)$ planets around very nearby and bright stars can in principal be achieved with long observation times at short wavelengths ( $\sim 0.6$ to $0.9 \mu \mathrm{m}$ ) with a photon-noise limited (ie. artifact free) instrument such as the proposed ZIMPOL concept on for the VLT Planet Finder (Gisler et al., 2004). Such measurements combine all the benefits of improved $\mathrm{AO}$ and advanced coronagraphy with far more accurate differential measurements of the planetary polarization signal. Indeed, polarized, reflected light from a planet is not a function of age, unlike intrinsic radiation.

3.3.3 Future systems (2015/2020). The potential of groundbased observations after 2015 will essentially depend on a number of technological developments which remain yet to be demonstrated. Reaching even higher contrasts than those foreseen for the coming generation of systems, in the $10^{8}$ to several $10^{10}$ range, ie. $\Delta m>25$, at small separations $(\sim$ 0.1 ") is motivated by the search for lower mass exoplanets, ultimately down to the direct detection of Earth analogs. Similar performance will be required also for fine characterization of previously detected EGPs, with higher SNR and higher spectral resolution, $R \sim 1000$, and very small signal variations along the planet orbit.

Reaching such extreme performances in contrast will definitively require very detailed system engineering, based on the experience obtained with the previous generations of instruments, and accounting for other, effects such as atmospheric scintillation (Masciadri et al., 2004). These observations will also require much larger collecting areas (ELT class) for the detection of terrestrial planets.

Both the development of larger telescopes and of new instruments of unprecedented precision appears very challenging but can be seen as realistic, with appropriate development plans and corresponding resources, on the $\sim 2015-$ 2020 time scale. Efforts are being made both in the US and in Europe to start the design of such telescopes (Thirty Meter Telescope, OWL) and corresponding instruments (PFI, EPICS). However, one should note that this new generation 
of facilities will certainly not extend the sample of accessible stellar targets. On the contrary, both the planet faintness and the even higher precision $\mathrm{AO}$ correction required will restrict the potential targets to the brightest stars.

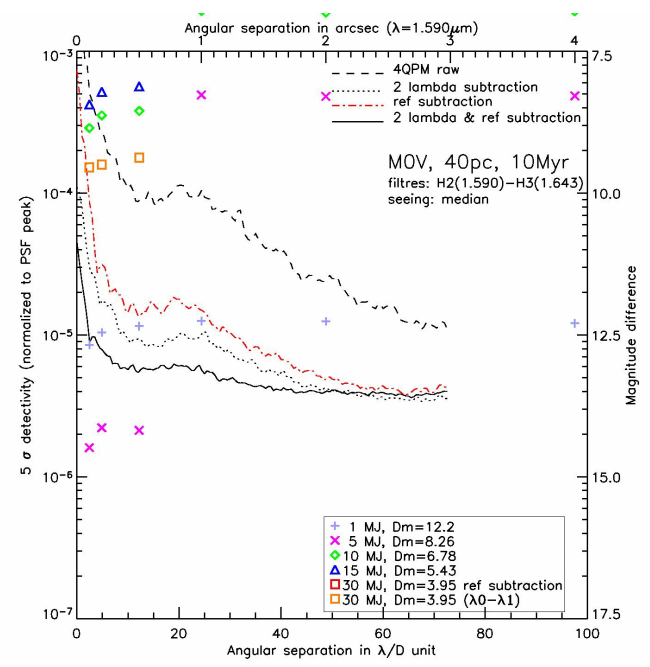

Fig. 4.- Expected performance of the VLT Planet Finder instrument for the detection of young planetary systems using the simultaneous differential imaging technique. The best performance is achieved in the $\mathrm{H}-$ band with the use of a reference star (double subtraction, i.e. 2-wavelength subtraction and residual pattern subtraction from the reference target observation). In this case the host star has an M0 type, is aged $10 \mathrm{Myr}$ and is located at $40 \mathrm{pc}$ from the Sun. Planets with $1 \mathrm{M}_{J}$ are detected in the $\mathrm{H} 2-\mathrm{H} 3$ filter pair (respectively 1.590 and $1.643 \mu \mathrm{m}$ ) beyond 0.15 ". Planets with $5 \mathrm{M}_{J}$ are easily detected, and spectro-photometry is feasible beyond 0.1 " (detection in all NIR filters) without a reference star.

Results on the lowest planetary masses (1 to 10 Earth masses) will be detections at relatively low SNR and low spectral resolution. Because a larger variety of atmospheric composition and physical parameters can be expected for terrestrial planets, further spectral diagnosis and higher SNR will be required with longer integration times for positive detections. Ultimately, the refined characterization of the atmosphere and the search for biomarkers will be even an order of magnitude more difficult. Indeed, specific known features (like $\mathrm{O}_{2}, \mathrm{NO}_{2}$, the vegetation red-edge signature, and others) often have only small absorption depths (requiring corresponding high SNR spectra), or are strongly blended with other absorption features, including our own atmospheric absorption. At this stage, a complete analysis in terms of complexity, cost, schedule and risk is required to compare the relative interests of observations from the ground or from space. For the ultimate performance goals, the fundamental limitations associated with atmosphere (for both image perturbation and absorption) will increasingly de-favor the ground-based approach even if larger collecting areas can be achieved.

\section{INTERFEROMETRY}

While adaptive optics methods have achieved incredible dynamic range for imaging beyond the first or second Airy ring of a telescope PSF, only long-baseline interferometers can directly search for planets orbiting parent stars within $\sim 100$ milli-arcseconds ( $1 \mathrm{AU}$ at $10 \mathrm{pc}$ ) - at least until the next generation of giant telescopes are built. While interferometers have a resolution advantage, they suffer from signal-to-noise disadvantages since collecting area is usually smaller, net optical throughput is low, and Poisson noise from unattenuated stellar light usually dominates the noise budget. Fortunately, planets in sub-AU orbits are considerably warmer than their more distant siblings, relaxing the dynamic-range requirement for direct detection. In this section, we will outline the current observational approaches being attempted for exoplanet detection.

From basic signal-to-noise considerations, Earth-mass planets are not realistically detectable with conventional interferometers based around 8m-class telescopes (or smaller ones). However, RV surveys have uncovered a diversity of planets with a range of masses and semi-major axes in nearby systems. Indeed, massive planets at $\lesssim 1 \mathrm{AU}$ distances are exciting to study from the perspective of comparative planetology since we lack solar system analogues. This includes the fascinating subset of planets known as "hot Jupiters", including the first exoplanet 51 Peg.

As a mechanism to focus discussion (and admit humility), we concentrate specifically on detectability of Hot Jupiters since they will prove easiest to detect. Hot Jupiters are typically 0.05-0.10 AU from their parent star with expected surface temperatures between 1000-1500K. Calculations (e.g. Guillot et al., 1996; Burrows et al., 1997; Goukenleuque et al., 2000; Sudarsky et al., 2000; Barman et al., 2001) indicate the planet-to-star flux ratio to be a $>10^{-4}$, a number which will only sound large to readers finishing the last section of this chapter. Fig. 5 shows an example of an early calculation by Seager et al. (2000) that suggests the relative ease of near-infrared detection. Note that these planets should exhibit a rich molecular spectrum dependent on atmospheric density and temperature structure, making even a low resolution spectrum measurement incredibly exciting.

\subsection{Narrow-angle astrometry}

The interferometric techniques discussed here are related to, but distinct from, "astrometric" search strategies which attempt to detect physical motion of the host star in the plane of the sky (e.g. Gatewood, 1976). This technique is analogous to the radial velocity methods and does not directly detect the planet itself (only the host star's "wobble"). The modern equivalent is called "narrow-angle astrometry" using special-purpose long-baseline optical interferometers built with a "dual-star" module to simultaneously observe a target and reference star. This technique has been well- 
studied, and is being pursued by Keck and VLT Interferometers and the Space Interferometry Mission. Recent exciting results have been reported for the Palomar Testbed Interferometer (Lane and Muterspaugh, 2004; Muterspaugh et al., 2005), achieving 10-100 micro-arcsecond relative astrometry between components of close (sub-arcsecond) binaries. Demonstrated performance is adequate to detect short-period Jupiter-mass planets if present in their (binary) sample.

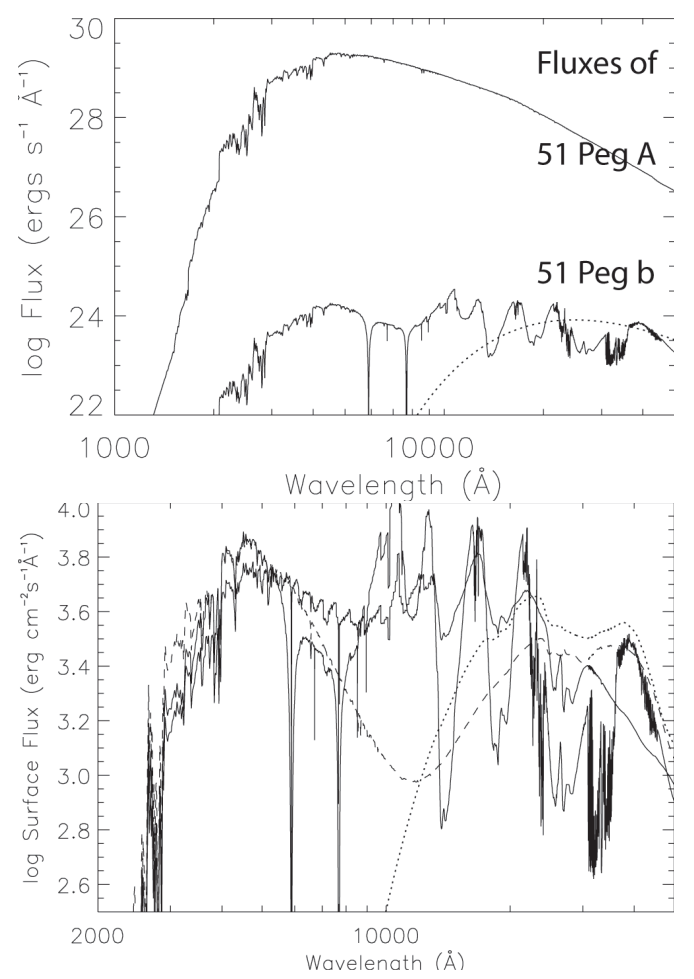

Fig. 5.- These figures show calculations of the emergent spectrum of 51 Peg b published by Seager et al. (2000) under various assumptions (their figures 3 and 2 respectively). The top panel explores different dust size distributions, while the bottom panel compares a fiducial model spectrum to that of the host star. The flux ratio is expected to be $\sim 10^{4}$ in the near-IR observing bands.

\subsection{Differential Phase}

The first "direct" interferometric detection technique we will discuss is called the "differential phase" method. It is a multi-wavelength approach to find massive exoplanets by detecting a very slight photocenter shift (of the star+planet system) between different infrared filters. This occurs because of wavelength-dependent flux ratios caused by molecular opacity in the planet's atmosphere and the large temperature difference between star and planet (i.e. the differential phase method; e.g. Akeson and Swain, 1999; Lopez and Petrov, 2000). Fig. 6 illustrates how an interferometer can be used to detect a faint companion (planet) to a star. In the left panel of this figure, an optical interferometer is represented as a Young's two-slit experiment (Born and Wolf, 1965). Flat wave fronts from a distant source impinge on the slits and produce an interference pattern on an illuminated screen. Multiple sources would produce multiple such fringe patterns, incoherently adding together in power. The right panel illustrates how the presence of a planet would cause a small reduction in fringe contrast (visibility) and would shift the fringe phase a correspondingly tiny amount.

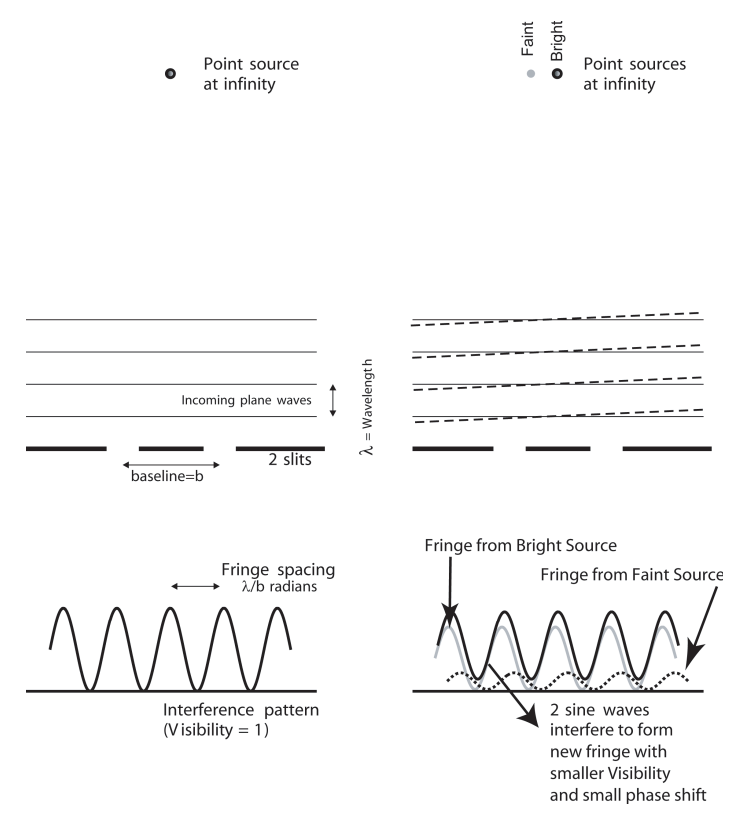

Fig. 6. - The figure illustrates how the presence of a planet causes a phase shift in the stellar fringe observed by a long-baseline optical interferometer.

In the limit of high brightness ratio (very appropriate here!), we can be more quantitative. Assuming a planetto-star brightness ratio of $\alpha=\frac{F_{\text {planet }}}{F_{*}}$, projected baseline $\vec{b}$, observing wavelength $\lambda$, and planet-star separation $\vec{\delta}$, then the normalized Complex Visibility $\tilde{V}$ observed by an interferometer is simply (assuming both components are not resolved by the interferometer):

$$
\tilde{V}=\frac{1+\alpha e^{-2 \pi i \frac{\vec{b}}{\lambda} \cdot \vec{\delta}}}{1+\alpha}
$$

In the limit of $\alpha<<1$ appropriate for exoplanet detection experiments, the last equation takes a simpler form when we consider the Visibility amplitude $|V|$ and phase $\Phi_{V}$ separately, $\tilde{V}=|V| e^{-i \Phi_{V}}$ :

$$
\begin{aligned}
& |V| \approx 1-2 \alpha \sin ^{2}\left(\pi \frac{\vec{b}}{\lambda} \cdot \vec{\delta}\right) \\
& \Phi_{V} \approx \alpha \sin \left(2 \pi \frac{\vec{b}}{\lambda} \cdot \vec{\delta}\right)
\end{aligned}
$$

Hence, by measuring $\Phi_{V}$ (or alternatively $|V|$ ) at various baselines $\vec{b}$ and wavelengths $\lambda$, both the brightness ratio spectrum $\alpha(\lambda)$ and the separation vector $\vec{\delta}$ between the star 
and planet can be determined. If the stellar photosphere is partially resolved (as will often be the case), the phase signal is boosted, easing detection. Unfortunately, the atmosphere at visible and infrared wavelengths defeats this simplistic approach, and this is detailed below.

We can see how telescope-specific phase delays caused by atmospheric turbulence (or anything else) affect the measured visibility amplitude and phase by considering the same idealized interferometer sketched again in Fig. 7. If the pathlength above one slit is changed (due to a pocket of warm air moving across the aperture, for example), the interference pattern will be shifted by an amount depending on the difference in pathlength of the two legs in this simple interferometer. If the extra pathlength is half the wavelength, the fringe pattern will shift by half a fringe, or $\pi$ radians. The phase shift is completely independent of the slit separation, and only depends on slit-specific phase delays. The corruption of this phase information has serious consequences. For the search for exoplanets, the tiny signal of the planet, as encoded by a baseline-dependent phaseshift, is lost.

But all is not lost: by comparing fringes at one wavelength to that at another, we can track the fluctuating atmosphere term and recover an estimate of the "differential phase." This method should be relatively insensitive to the atmospheric turbulence. This method also benefits from the bright flux of the target star, a beacon through the changing atmosphere acting much like a natural guide star in adaptive optics. However, recent studies of line-of-sight variability of atmospheric water vapor (Colavita et al., 2004) indicate that differential chromatic dispersion might be more difficult to calibrate for differential phase methods than originally expected.

We note in passing that atmospheric turbulence affects the calibration of visibility amplitudes as well, since phase irregularities over each telescope aperture degrade spatial coherence. Calibrating visibility amplitudes to the precision needed for detecting planets is considered even more difficult than phase methods (due to poorer statistical properties), although it has been discussed (Coudé du Foresto et al., 2000).

Table 1 summarize the current active efforts to detect exoplanets using differential phase

\subsection{Precision Closure Phase Method}

There is another interferometry technique which is robust to both atmospheric phase shifts and also differential chromatic dispersion. Consider the right panel of Fig. 7 in which a phase delay is introduced above telescope 2 in a 3-telescope interferometer. This causes a phase shift in the fringe detected between telescopes 1-2, as discussed in the last section. Note that a phase shift is also induced for fringes between telescopes 2-3; however, this phase shift is equal but opposite to the one for telescopes 1-2. Hence, the sum of three fringe phases, between 1-2, 2-3, and 3-1, is insensitive to the phase delay above telescope 2 . This argument holds for arbitrary phase delays above any of the three telescopes. In general, the sum of three phases around a closed triangle of baselines, the closure phase, is a good interferometric observable; that is, it is independent of telescope-specific phase shifts induced by the atmosphere or optics.

The idea of closure phase was first introduced to compensate for poor phase stability in early radio VLBI work (Jennison, 1958). Application at higher frequencies was first mentioned by Rogstad (1968), but only much later carried out in the visible/infrared through aperture masking experiments (Baldwin et al., 1986; Haniff et al., 1987; Readhead et al., 1988). Currently six separate-element interferometers have reported obtaining closure phase measurements, in the optical (visible/infrared), first at the Cambridge Optical Aperture Synthesis Telescope (COAST; Baldwin et al., 1996), followed by the Navy Prototype Optical Interferometer (NPOI; Benson et al., 1997), the Infrared Optical Telescope Array (IONIC3 combiner, Monnier et al., 2004b), the Infrared Spatial Interferometer (ISI, Weiner et al., 2006), VLT Interferometer (AMBER combiner), and most recently at the CHARA Interferometer (MIRC combiner).
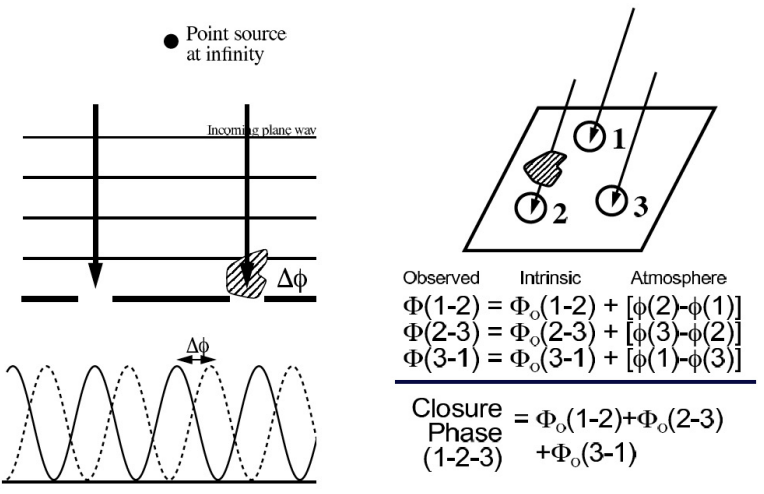

Fig. 7.- (left panel): In an interferometer, a phase delay above an aperture causes a phase shift in the detected fringe pattern. (right panel): Phase errors introduced at any telescope causes equal but opposite phase shifts, canceling out in the closure phase (after Readhead et al., 1988)

While differential-phase methods have been the focus of much current activity, precision measurements of only closure phases can also be used to detect faint companions. As discussed, the closure phase is formed by summing the interferometer phases on three baselines around a triangle of telescopes, and this quantity is immune to atmospheric phase delays. Ségransan et al. (2000) and Monnier (2002) recently discussed how closure phases are immune to dominant calibration problems of differential phase and that they can also be used to solve for all the parameters of a binary system without needing to measure any visibility amplitudes. Fig. 8 shows the expected closure phase signature of the hot Jupiter $v$ And $\mathrm{b}$, one of the best candidates for direct detection, as it would appear to the CHARA Interfer- 
ometer.

The derivation of the planet-induced fringe phase (last section) can be used to prove that the closure phase yields an observable with the same magnitude as differential phase methods, when the planet-star separation is clearly resolved. Unfortunately, the closure phase amplitude scales like baseline to the third power for short baselines, making detections of partially-resolved systems difficult or impossible. Note that differential phase scales linearly with baseline in this case and thus remains a viable planet detection even for relatively short baselines (e.g., the 85-m Keck-Keck baseline).

Table 2 contains the list of hot Jupiter systems, planets with semi-major axes within $0.1 \mathrm{AU}$ of their parent stars. Included in this table are important observing parameters and one can see a few favorable targets with very bright infrared magnitudes (e.g., $\mathrm{K} \sim 4$ ). Also, find in the last column of the table the minimum baseline needed to resolve known hot Jupiters at $1.65 \mu \mathrm{m}$. Essentially, all of these sources can be resolved by VLTI and CHARA interferometers (longest baseline 220-m and 330-m respectively), thus permitting precision closure phase work as well as differential phase studies.

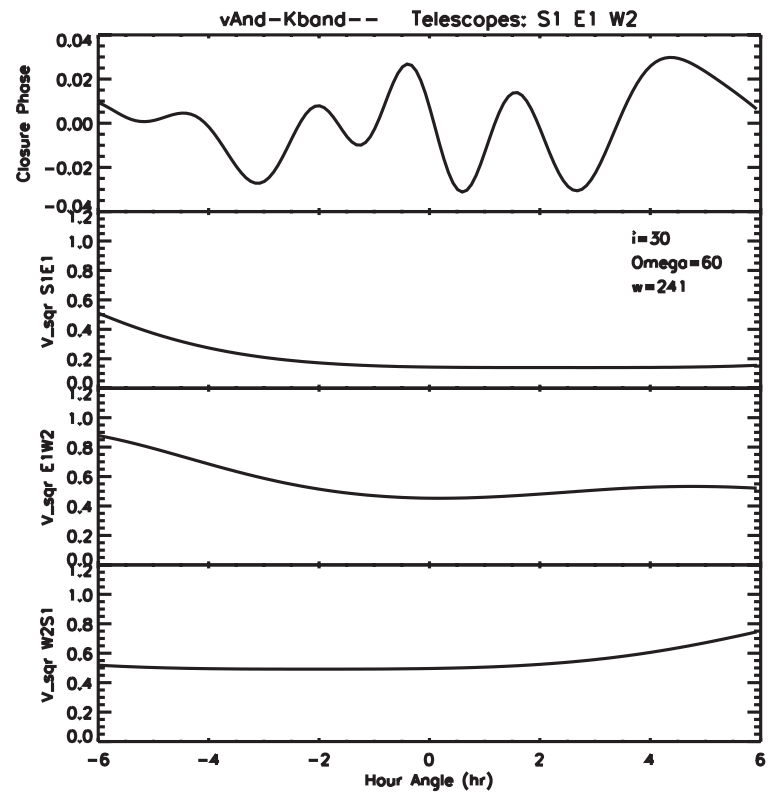

Fig. 8. - The top panel shows the predicted closure phase signal for CHARA interferometer observations of $v$ And $b$. The closure phase is significantly boosted because the star itself is partially-resolved by the $300-\mathrm{m}$ baselines of this interferometer (see bottom 3 panels showing the predicted visibility-squared for this observation).

Current published measurement precision of closure phases is only 0.3 to 5 degrees (Tuthill et al., 2000; Benson et al., 1997; Young et al., 2000; Millour et al., 2006); for reference, a typical closure phase for a binary with brightness ratio of $10^{4}$ is $\sim 0.01$ degrees. Recently, IOTA interferometer has demonstrated a calibration stability of 0.1 degrees in closure phase using the IONIC combiner, an integrated optics device that minimizes drifts by minia- turizing the waveguides (Berger et al., 2003). Improving the three orders of magnitudes needed to detect even the brightest possible exoplanet is a daunting challenge. Fig. 9 shows the results from a recent study of closure phase stability at the CHARA Interferometer, using the new MIRC combiner (Monnier et al., 2004a). These data were taken under very poor seeing on the very first night of CHARA closure phases, thus significantly better results should be straightforward to achieve. The closure phase here appears stable over 2 hours with no detectable drifts. The formal closure phase error for the average of the whole dataset is 0.03 degrees, nearly sufficient to detect an extrasolar planet (see Fig. 8). While there are surely unconsidered systematic effects (perhaps due to birefringence or drifts in optical alignment) which will degrade the sensitivity of the precision closure phase technique, the lack of any "showstopper" effects, like differential atmospheric dispersion for the differential phase methods, strongly motivates current efforts.

\subsection{Nulling Interferometry}

Another approach to detecting exoplanets is nulling interferometry. First demonstrated by Hinz et al. (1998) on the MMT telescope, a nulling interferometer introduced an extra (achromatic) phase delay in one arm of the interferometer so that light from the central star destructively interferes. Since the null depends on the incident angle of the starlight, one can tune the interferometer to selectively null out the star while allowing light from the planet to still be measured by the interferometer. More details can be found in Serabyn et al. (2000). A nuller is the interferometryequivalent of a coronagraph on single-aperture telescope, minimizing noise from Poisson fluctuations of stellar light and relaxing the calibration requirements on the dynamic range.

Nulling interferometry is very difficult to carry-out from the ground because of atmospheric turbulence. Not only do the path lengths have to matched, requiring active tracking of piston fluctuations at the nm-level precision, but the wave front across each telescope must also be corrected using adaptive optics in order to match the amplitude of the fringes too. For these reasons, significant gains using nulling from the ground is only expected at longer, midinfrared wavelengths. Because nulling interferometry is the foundation of all space-based interferometers aiming to find Earth-like planets, ground-based projects are focused on technology development and measuring zodiacal dust properties of future observing targets (exo-zodiacal dust emission may be the dominant background noise source for Earth-mass planet detection using nulling).

The Keck Interferometer project (NASA) is beginning a survey of nearby stars using nulling interferometry (in the thermal infrared), and first results of 100-to-1 nulls on the sky were recently reported (Colavita, private communication). The explicit aim of this survey is not to discover exoplanets, but rather to measure the exozodiacal light in the thermal infrared. However, it has been suggested that 
the survey strategy will also be sensitive to close-in Jupitermass planets (Kuchner, private communication) if present. The VLTI originally was to have used the GENIE instrument, a nuller testbed optimized for $2-5 \mu \mathrm{m}$, for a zodiacal light and faint companion survey at somewhat shorter wavelengths. However, the ultimate fate for GENIE is still being considered and it is currently not known if this instrument will see sky-time at the VLTI or anywhere. Lastly, the Large Binocular Telescope Interferometer (LBTI) has ambitious plans for mid-infrared nulling in order to map zodiacal dust and search for faint companions. First light for the LBTI is a number of years away, but the project is rapidly progressing.

\subsection{Immediate Outlook}

Excitement in the interferometry field is motivated by the convergence of many developments: newly-commissioned large-aperture and long-baseline interferometers (VLTI, Keck, CHARA), new combiner architectures optimized for precision calibration of (closure) phases (AMBER, IONIC3, MIRC), and the recent discovery of bright hot Jupiter systems that should be "easy" to detect (51 Peg, $\tau$ Boo, $v$ And). A back-of-the-envelope calculation for CHARA will further motivate and justify our optimism and excitement. We know from Table 2 that the CHARA interferometer has sufficiently long baselines to resolve all the planet-star separations listed. However, it is not obvious if CHARA has the light collecting capability to detect such a small phase shift in the closure phase. Assuming a net CHARA optical efficiency of $5 \%, H=4$ mag (e.g., $51 \mathrm{Peg}$ ), bandwidth $0.05 \mu \mathrm{m}, 1 \mathrm{~m}$ telescopes, and coherent integration time of $0.1 \mathrm{sec}$, we will detect $\sim 5 \times 10^{4}$ photons per telescope per coherence time. Depending on the exact beam combination strategy, each $0.1-\mathrm{sec}$ fringe measurement should have a SNR 200, yielding a closure phase measurement with about $\sim 0.50$ degrees $\left(\sqrt{3} \frac{1}{200} \mathrm{rad}\right)$. Improving this to 0.0011 degrees (e.g., 5- $\sigma$ detection of $10^{-4}$ companion) would require an additional 10000 measurements (total on-source integration time of $\sim 30$ minutes). Thus, it is theoretically possible to measure the lowresolution spectrum of the 51-Peg companion with less than a night of integration time with 1-m telescopes, if precision calibration schemes can be developed.

Currently, the biggest limitation of today's systems is low optical throughput. The above calculation assumed $5 \%$ throughput, while measured values are $3-10 \times$ worse depending on seeing. For most current interferometers, optical throughput is $\sim 1 \%$ and efforts are being concentrated on improving this performance. While large aperture telescope arrays like VLTI and Keck have plenty of aperture to overcome these losses, fairly limited observing time is allocated to interferometry since these telescopes are used by the general astronomy community.

Table 1 summarizes all the major efforts to detect exoplanets using current and planned interferometers, with special emphasis on current ground-based projects. Most likely, the first detection will come from the innovative AMBER combiner on the Very Large Telescope Interferometer within the next 2 years. However, there is intense competition at many of the world's interferometers, and so keep your eyes open for new and unexpected discoveries.

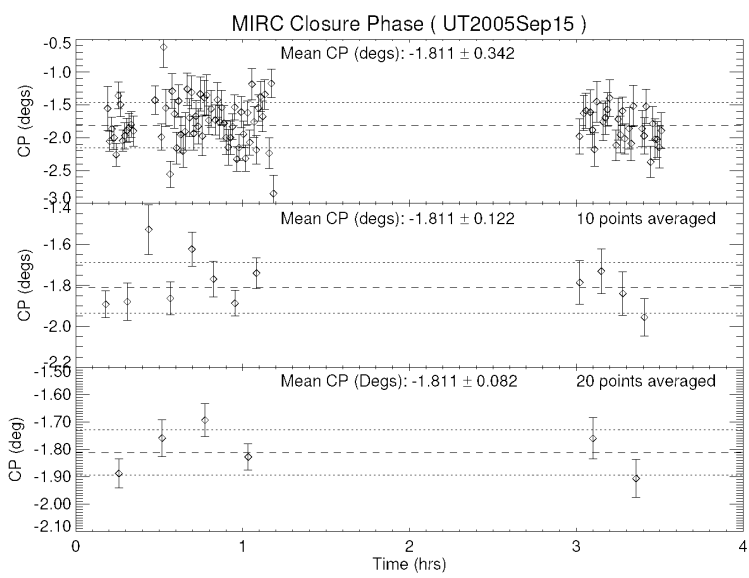

Fig. 9.- This figure shows the results of a closure phase stability test at the CHARA interferometer under very poor seeing conditions using the MIRC Combiner. This dataset currently represents the highestprecision closure phase ever achieved on a star - corresponding to \pm 0.03 in $\sim 2$ hours. In the top panel, each point is a 45 -sec average for observations of test source $\beta$ Tau. The middle panel shows averages of 10 points while the bottom panel shows the results for 20 data points. The final precision is within about an order-of-magnitude of that required for planet detection.

Acknowledgments. JDM thanks Ming Zhao for his help in making some of the figures in this review. JLB and DM are very gratefull to the VLT "Planet Finder" Phase A study team, and especially A.-M. Lagrange and C. Moutou, who greatly contributed to the discussion and results presented in this paper. BRO thanks his collaborators on the Lyot Project for their dedication and hard work.

\section{REFERENCES}

Aime C. and Soummer R. (2004) Astrophys. J., 612, L85-L88.

Akeson R. L. and Swain M. R. (1999) In Working on the Fringe: Optical and IR Interferometry from Ground and Space (S. C. Unwin and R. V. Stachnik, eds.), pp. 89-94. ASP Conference Series, San Francisco.

Angel R. and Burrows A. (1995) Nature, 374, 678-679.

Arnold L. and Schneider J. (2004) Astron. Astrophys., 420, 11531162.

Baldwin J. E., Haniff C. A., Mackay C. D., and Warner P. J. (1986) Nature, 320, 595-597.

Baldwin J. E., Beckett M. G., Boysen R. C., Burns D., Buscher D. F., Cox G. C., Haniff C. A., Mackay C. D., Nightingale N. S., Rogers J., Scheuer P. A. G., Scott T. R., Tuthill P. G., Warner P. J., Wilson D. M. A., and Wilson R. W. (1996) Astron. Astrophys., 306, L13-L16.

Barman T. S., Hauschildt P. H., and Allard F. (2001) Astroph. J., 556, 885-895.

Baraffe I., Chabrier G., Allard F., and Hauschildt P. (2002) Astron. Astrophys., 382, 563-572. 
Baudoz P., Boccaletti A., Rabbia Y., and Gay J. (2005) Publ. Astron. Soc. Pac., 117, 1104-1111.

Beaulieu J.-P., Bennett D. P., Fouqué P., Williams A., Dominik M., Jorgensen U. G., Kubas D. et al. (2006) Nature, 439, 437-440.

Benson J., Hutter D., Elias N. M. I., Bowers P. F., Johnston K. J., Hajian A. R., Armstrong J. T., Mozurkewich D., Pauls T. A., Rickard L. J., Hummel C. A., White N. M., Black D., and Denison C. (1997) Astron. J., 114, 1221-1226.

Berger J.-P., Haguenauer P., Kern P. Y., Rousselet-Perraut K., Malbet F., Gluck S., Lagny L., Schanen-Duport I., Laurent E., Delboulbe A., Tatulli E., Traub W. A., Carleton N., Millan-Gabet R., Monnier J. D., and Pedretti E. (2003) In Proceedings SPIE, 4838 (W. A. Traub, ed.), pp. 1099-1106. The International Society for Optical Engineering, Bellingham.

Berton A., Feldt M., Gratton R., Hippler S., and Henning T. (2006) New Astronomy Reviews, 49, 661-669.

Beuzit J.-L., Chauvin G., Delfosse X., Forveille T., Lagrange A.M., Marchal L., Mayor M., Ménard F., Mouillet D., Perrier C., Ségransan D., and Udry S. (2004) In Astronomy with High Contrast Imaging (C. Aime and R. Soummer, eds), pp. 319336. EAS Publications Series, Paris.

Beuzit J.-L., Feldt M., Mouillet D., Moutou C., Dohlen K., Puget P., Fusco T. et al. (2006) In Direct Imaging of Exoplanets: Science and Techniques, Proceedings of IAU Colloqium, 200 (C. Aime and F. Vakili, eds.), pp. 317-322. Cambridge University Press, Cambridge.

Bloemhof E. E., Dekany R. G., Troy M., and Oppenheimer B. R. (2001) Astrophys. J., 558, L71-L76.

Bonfils X., Forveille T., Delfosse X., Udry S., Mayor M., Perrier C., Bouchy F., Pepe F., Queloz D., and Bertaux J.-L. (2005) Astron. Astrophys., 443, L15-L18.

Born M. and Wolf E. (1965) Principles of optics. Electromagnetic theory of propagation, interference and diffraction of light, $3 \mathrm{rd}$ edition.

Brandeker A., Jayawardhana R., and Najita J. (2003) Astron. J., 126, 2009-2014.

Brown, R. A. (2005) Astrophys. J., 624, 1010-1024.

Burrows A. (2005) Nature, 433, 261-268.

Burrows A. and Sharp C. M. (1999) Astrophys. J., 512, 843-863.

Burrows A., Marley M., Hubbard W. B., Lunine J. I., Guillot T., Saumon D., Freedman R., Sudarsky D., and Sharp C. (1997) Astrophys. J., 491, 856-875.

Butler R. P., Marcy G. W., Fischer D. A., Brown T. M., Contos A. R., Korzennik S. G., Nisenson P., and Noyes R. W. (1999) it Astrophys. J., 526, 916-927.

Butler R. P., Vogt S. S., Marcy G. W., Fischer D. A., Wright J. T., Henry G. W., Laughlin G., and Lissauer J. J. (2004) Astrophys. J., 617, 580-588.

Chabrier G., Baraffe I., Allard F., and Hauschildt P. (2000) Astrophys. J., 542, 464-472.

Chauvin G., Ménard F., Fusco T., Lagrange A.-M., Beuzit J.-L., Mouillet D., and Augereau J.-C. (2002) Astron. Astrophys., 394, 949-956.

Chauvin G., Lagrange A.-M., Dumas C., Zuckerman B., Mouillet D., Song I., Beuzit J.-L., and Lowrance P. (2005a) Astron. Astrophys., 438, L25-L28.

Chauvin G., Lagrange A.-M., Zuckerman B., Dumas C., Mouillet D., Song I., Beuzit J.-L., Lowrance P., and Bessell M. S. (2005b) Astron. Astrophys., 438, L29-L32.

Clénet Y., Kasper M., Ageorges N., Lidman C., Fusco T., Marco O., Hartung M., Mouillet D., Koehler B., Rousset G., and Hubin N. (2004) In Proceedings SPIE, 5490 (D. B. Calia, B. L.
Ellerbroek and R. Ragazzoni, eds.), pp. 107-117. The International Society for Optical Engineering, Bellingham.

Close L. M., Lenzen R., Guirado J. C., Nielsen E. L., Mamajek E. E., Brandner W., Hartung M., Lidman C., and Biller B. (2005) Nature, 433, 286-289.

Colavita M. M., Swain M. R., Akeson R. L., Koresko C. D., and Hill R. J. (2004) Publ. Astron. Soc. Pac., 116, 876-885.

Coudé du Foresto V. (2000) In From exoplanets to Cosmology: The VLT Opening Symposium (J. Bergeron and A. Renzini, eds.), pp. 560. Proceedings of the ESO Symposium, SpringerVerlag, Berlin.

Dekany R. G., Nelson J. E., and Bauman B. J. (2000) In Proceedings SPIE, 4003 (P. Dierickx, ed.), pp. 212-225. The International Society for Optical Engineering, Bellingham.

Dekany R. G. (2004) In Proceedings SPIE, 5382 (G. E. Jabbour and J. T. Rantala, eds.), pp. 12-20. The International Society for Optical Engineering, Bellingham.

Ftaclas C., Martin E. L., and Toomey D. (2003) In Brown Dwarfs, Proceedings of IAU Symposium, 211 (E. Martin, ed.), pp. 521. Astronomical Society of the Pacific, San Francisco.

Fusco T., Rousset G., Beuzit J.-L., Mouillet D., Dohlen K., Conan R., Petit C., and Montagnier G. (2005) In Proceedings SPIE, 5903 (R. K. Tyson and M. Lloyd-Hart, eds.), pp. 178-189. The International Society for Optical Engineering, Bellingham.

Gatewood G. (1976) Icarus, 27, 1-12.

Gisler D., Schmid H. M., Thalmann C. P., Hans P., Stenflo J. O., Joos F., Feldt M. et al. (2004) In Proceedings SPIE, 5492 (A. F. M. Moorwood and I. Masanori, eds.), pp. 463-474. The International Society for Optical Engineering, Bellingham.

Goukenleuque C., Bézard B., Joguet B., Lellouch E., and Freedman R. (2000) Icarus, 143, 308-323.

Gould A. and Loeb A. (1992) Astrophys. J., 396, 104-114.

Guillot T. (1999) Science, 286, 72-77.

Guillot T., Burrows A., Hubbard W. B., Lunine J. I., and Saumon D.(1996) Astrophys. J., 459, L35-L38.

Guyon O., Pluzhnik E. A., Galicher R., Martinache F., Ridgway S. T., and Woodruff R. A. (2005) Astrophys. J., 622, 744-758.

Han I., Black D. C., and Gatewood G. (2001) Astrophys. J., 548, L57-L60.

Haniff C. A., Mackay C. D., Titterington D. J., Sivia D., and Baldwin J. E. (1987) Nature, 328, 694-696.

Hinz P. M., Angel J. R. P., Hoffmann W. F., McCarthy D. W., McGuire P. C., Cheselka M., Hora J. L., and Woolf N. J. (1998) Nature, 395, 251-253.

Jennison R. C. (1958) Mon. Not. R. Astron. Soc., 118, 276-284.

Jha S., Charbonneau D., Garnavich P. M., Sullivan D. J., Sullivan T., Brown T. M., and Tonry J. L. (2000) Astrophys. J., 540, L45-L48.

Kaltenegger L. and Fridlund M. (2005) Advances in Space Research, 36, 1114-1122.

Kasting J. F. and Catling D. (2003) Ann. Rev. Astron. Astrophys., 41, 429-463.

Lane B. F. and Muterspaugh M. W. (2004) Astrophys. J., 601, 1129-1135.

Lenzen R., Close L., Brandner W., Biller B., and Hartung M. (2004) In Proceedings SPIE, 5492 (A. F. M. Moorwood and I. Masanori, eds), pp. 970-977. The International Society for Optical Engineering, Bellingham.

Lopez B. and Petrov R. G. (2000) In From exoplanets to Cosmology: The VLT Opening Symposium (J. Bergeron and A. Renzini, eds), pp. 565. Proceedings of the ESO Symposium, Springer-Verlag, Berlin. 
Lyot, B. (1939) Mon. Not. R. Astron. Soc., 99, 538-580.

Macintosh B. A., Olivier S. S., Bauman B. J., Brase J. M., Carr E., Carrano C. J., Gavel D. T., Max C. E., and Patience J. (2002) In Proceedings SPIE, 4494 (R. K. Tyson, D. Bonaccini and M. C. Roggemann, eds), pp. 60-68. The International Society for Optical Engineering, Bellingham.

Macintosh B. A., Bauman B., Wilhelmsen E. J., Graham J. R., Lockwood C., Poyneer L., Dillon D., Gavel D. T. et al. (2004) In Proceedings SPIE, 5490 (D. Bonaccini, B. L. Ellerbroek and R. Ragazzoni, eds), pp. 359-369. The International Society for Optical Engineering, Bellingham.

Mao S. and Paczynski B. (1991) Astrophys. J., 374, L37-L40.

Marcy G. W., Butler R. P., Fischer D. A., and Vogt S. S. (2003) In Scientific Frontiers in Research on exoplanets (D. Deming and S. Seager, eds), pp. 1-16. ASP Conference Series, San Francisco.

Masciadri E., Feldt M., and Hippler S. (2004) Astrophys. J., 613, 572-579.

Masciadri E., Mundt R., Henning T., Alvarez C., and Barrado y Navascués D. (2005) Astrophys. J., 625, 1004-1018.

Mayor M. and Queloz D. (1995) Nature, 378, 355-359.

Mennesson B., Léger A., and Ollivier M. (2005) Icarus, 178, 570588.

Millour F. et al. (2006) astro-ph/0512245.

Monnier J. D. (2002) In Eurowinter School: Observing with the Very Large Telescope Interferometer (G. Perrin and F. Malbet, eds.), pp. 213-226.

Monnier J. D., Berger J.-P., Millan-Gabet R., and Ten Brummelaar T. A. (2004a) In Proceedings SPIE, 5491 (W. A. Traub, ed.), pp. 1370. The International Society for Optical Engineering, Bellingham.

Monnier J. D., Traub W. A., Schloerb F. P., Millan-Gabet R., Berger J.-P., Pedretti E., Carleton N. P., Kraus S., Lacasse M. G., Brewer M., Ragland S., Ahearn A., Coldwell C., Haguenauer P., Kern P., Labeye P., Lagny L., Malbet F., et al. (2004b) Astrophys. J., 602, L57-L60.

Mouillet D., Beuzit J.-L., Chauvin G., and Lagrange A.-M. (2002) In Scientific Drivers for ESO Future VLT/VLTI Instrumentation (J. Bergeron and G. Monnet, eds), pp. 258-263. Proceedings of the ESO Workshop, Springer-Verlag, Berlin.

Muterspaugh M. W., Lane B. F., Konacki M., Burke B. F., Colavita M. M., Kulkarni S. R., and Shao M. (2005) Astron. J., 130, 2866-2875.

Neuhäuser R., Guenther E., Alves J., Húelamo N., Ott T., and Eckart A. (2003) Astronomische Nachrichten, 324, 535-542.

Neuhäuser R., Guenther E. W., Wuchterl G., Mugrauer M., Bedalov A., and Hauschildt P. H. (2005) Astron. Astrophys., 435, L13-L16.

Neuhäuser R. (2006) astro-ph/0509906, in press.

Oppenheimer B. R., Kulkarni S. R., Matthews K., and Nakajima T. (1995) Science, 270, 1478-1481.

Oppenheimer B. R., Kulkarni S. R., Matthews K., and van Kerkwijk M. H. (1998), Astrophys. J., 502, 932-941.

Oppenheimer B. R., Sivaramakrishnan A., and Makidon R. B. (2003) in The Future of Small Telescopes In The New Millennium. Volume III - Science in the Shadows of Giants (T. D. Oswalt, ed.) pp. 155-169. Astrophysics and Space Science Library, Kluwer Academic Publishers, Dordrecht.

Oppenheimer B. R., Digby A. P., Newburgh L., Brenner D., Shara M., Mey J., Mandeville C., Makidon R. B., Sivaramakrishnan A., Soummer R., Graham J. R., Kalas P., Perrin M. D., Roberts L. C. Jr., Kuhn J. R., Whitman K., Lloyd J. P. (2004) in Proceedings SPIE, 5490 (D. B. Calia, B. L. Ellerbroek and R. Ragazzoni, eds.), pp. 433-442. The International Society for Optical Engineering, Bellingham.

Readhead A., Nakajima T., Pearson T., Neugebauer G., Oke J., and Sargent W. (1988) Astron. J., 95, 1278-1296.

Rivera E. J., Lissauer J. J., Butler R. P., Marcy G. W., Vogt S. S., Fischer D. A., Brown T. M., Laughlin G., and Henry G. W. (2005) Astrophys. J., 634, 625-640.

Rogstad D. H. (1968) Applied Optics, 7, 585.

Seager S., Whitney B. A., and Sasselov D. D. (2000) Astrophys. J., 540, 504-520.

Ségransan D., Beuzit J.-L., Delfosse X., Forveille T., Mayor M., Perrier-Bellet C., and Allard F. (2000) In Proceedings SPIE, 4006 (P. Léna and A. Quirrenbach, eds.), pp. 269-276. The International Society for Optical Engineering, Bellingham.

Segura A., Kastin J. F., Meadows V., Cohen M., Scalo J., Crisp D., Butler R. A. H., and Tinetti G. (2005) Astrobiology, 5, 706-725.

Serabyn E., Colavita M. M., and Beichman C. A. (2000), In Thermal Emission Spectroscopy and Analysis of Dust, Disks, and Regoliths (M. L. Sitko, A. L. Sprague, and D. K. Lynch, eds.), pp. 357-365. ASP Conference Series, San Francisco.

Sivaramakrishnan A., Koresko C. D., Makidon R. B., Berkefeld T., and Kuchner M. J. (2001) Astrophys. J., 552, 397-415.

Soummer R., Dohlen K., and Aime C. (2003) Astron. Astrophys., 403, 369-381.

Soummer R. (2005) Astrophys. J., 618, L161-L164.

Sozzetti A. (2005) Publ. Astron. Soc. Pac., 117, 1021-1048.

Stoesz J. A., Véran J.-P., Rigaut F. J., Herriot G., Jolissaint L., Frenette D., Dunn J., and Smith M. (2004) In Proceedings SPIE, 5490 (D. B. Calia, B. L. Ellerbroek and R. Ragazzoni, eds.), pp. 67-78. The International Society for Optical Engineering, Bellingham.

Sudarsky D., Burrows A., and Pinto, P. (2000) Astrophys. J., 538, 885-903.

Trauger J. T., Burrows C., Gordon B., Green J. J., Lowman A. E., Moody D., Niessner A. F., Shi F., and Wilson D. (2004) In Proceedings SPIE, 5487 (A. Stohr, D. Jager and S. Iezekiel, eds.), pp. 1330-1336. The International Society for Optical Engineering, Bellingham.

Tuthill P. G., Monnier J. D., Danchi W. C., Wishnow E. H., and Haniff C. A. (2000) Publ. Astron. Soc. Pac., 112, 555-565.

van Dam M. A., Le Mignant D., and Macintosh B. A. (2004) Applied Optics, 43, 5458-5467.

Weiner J., Tatebe K., Hale D. D. S., Townes C. H., Monnier J. D., Ireland, M., Tuthill P. G., Cohen R., Barry R. K., Rajagopal J., and Danchi W. C. (2006) Astrophys. J., 636, 1067-1077.

Woolf N. J., Smith P. S., Traub W. A., and Jucks K. W. (2002) Astrophys. J., 574, 430-433.

Young J. S., Baldwin J. E., Boysen R. C., Haniff C. A., Lawson P. R., Mackay C. D., Pearson D., Rogers J., Saint.-Jacques D., Warner P. J., Wilson D. M. A., and Wilson R. W. (2000) Mon. Not. R. Astron. Soc., 315, 635-645.

Zuckerman B. and Song I. (2004) Ann. Rev. Astron. Astrophys., $42,685-721$. 
Table 1: Exoplanet Searches using Interferometry

\begin{tabular}{|c|c|c|}
\hline Interferometer & Method & Goals \\
\hline \multirow[t]{2}{*}{ CHARA Interferometer } & Precision/Differential Closure Phases & Measure low-res spectrum of hot Jupiters in $\mathrm{H} / \mathrm{K}$ bands \\
\hline & Precision Visibilities & Hot Jupiter detection using FLUOR combiner \\
\hline \multirow[t]{3}{*}{ Keck Interferometer } & Differential Phase ${ }^{a}$ & Hot Jupiter detection using $1-5 \mu \mathrm{m}$ wavelength range \\
\hline & Nulling & Mid-infrared search for zodiacal dust might uncover close-in planet \\
\hline & Narrow-Angle Astrometry (I) & Planet detection using Keck-Keck and outrigger array ${ }^{b}$ \\
\hline Navy Prototype Interferometer & Imaging & Detect hot Jupiter transit by imaging stellar photosphere in visible \\
\hline Palomar Testbed Interferometer & Narrow-Angle Astrometry & Detect massive planets around sub-arcsecond binaries \\
\hline \multirow[t]{4}{*}{ Very Large Telescope Interferometer } & Differential Phase & Low-res spectrum of hot/warm exoplanets (H/K bands) \\
\hline & Differential Closure Phase & Hot Jupiters and perhaps more difficult planets \\
\hline & Narrow-Angle Astrometry & PRIMA $^{c}$ instrument will allow long-term astrometry \\
\hline & Nulling $^{d}$ & GENIE instrument meant to detect low-mass companions (K,L,M bands) \\
\hline \multicolumn{3}{|c|}{ Future Plans } \\
\hline Antarctic Plateau Interferometer & Differential Phase/Closure Phase & Hot Jupiter planet characterization $(3-5 \mu \mathrm{m})$ \\
\hline Darwin (ESA) & Nulling Space Interferometer & Terrestrial Planet Finding (mid-infrared) \\
\hline Large Binocular Telescope Interferometer & Nulling & Detect (warm) massive exoplanets in thermal IR \\
\hline Space Interferometry Mission (NASA) & Astrometry & Detect low-mass exoplanets via induced planet wobble \\
\hline Terrestrial Planet Finder Interferometer (NASA) & Nulling Space Interferometer & Terrestrial Planet Finding (mid-infrared) \\
\hline
\end{tabular}

Table 2: Target List of (Nearby) exoplanets with Semi-major Axes $\lesssim 0.1$ AU (* denotes a favorable target)

\begin{tabular}{|c|c|c|c|c|c|c|c|c|c|}
\hline $\begin{array}{l}\text { Star } \\
\text { Name }\end{array}$ & $\begin{array}{l}\text { RA } \\
(\mathrm{J} 2000)\end{array}$ & $\begin{array}{l}\text { Dec } \\
(\mathrm{J} 2000)\end{array}$ & $\begin{array}{l}\text { Spectral } \\
\text { Type }\end{array}$ & $\begin{array}{l}\text { Distance } \\
(\mathrm{pc})\end{array}$ & $\begin{array}{l}\text { Semi-Major Axis } \\
\text { AU (mas) }\end{array}$ & $\begin{array}{l}\mathrm{M} \operatorname{sini} \\
\left(\mathrm{M}_{\mathrm{Jup}}\right)\end{array}$ & $\begin{array}{l}\mathrm{V} \\
\mathrm{mag}\end{array}$ & $\begin{array}{l}\mathrm{K} \\
\mathrm{mag}\end{array}$ & $\begin{array}{l}\text { Minimum }^{(a)} \\
\text { Baseline (m) }\end{array}$ \\
\hline HD $83443 \mathrm{~b}$ & 093711.8281 & -431619.939 & K0V & 43.5 & $0.038(0.87)$ & 0.35 & 8.24 & $\sim 6.3$ & 195 \\
\hline HD $46375 \mathrm{~b}$ & 063312.6237 & +052746.532 & K1IV & 33.4 & $0.041(1.23)$ & 0.249 & 7.84 & $\sim 5.8$ & 139 \\
\hline HD 179949 b & 191533.2278 & -241045.668 & F8V & 27.0 & $0.045(1.67)$ & 0.84 & 6.25 & $\sim 4.9$ & 102 \\
\hline HD $187123 b$ & 194658.1130 & +342510.288 & G5 & 47.9 & $0.042(0.88)$ & 0.52 & 7.86 & $\sim 6.3$ & 194 \\
\hline$* \tau$ Boo b & 134715.7429 & +172724.862 & F6IV & 15.6 & $0.0462(2.96)$ & 3.87 & 4.50 & $\sim 3.3$ & 57 \\
\hline HD $75289 b$ & 084740.3894 & -414412.452 & G0Ia & 28.9 & $0.046(1.59)$ & 0.42 & 6.36 & $\sim 5.0$ & 107 \\
\hline HD $209458 \mathrm{~b}$ & 220310.8 & +185304 & G0V & 47.1 & $0.045(0.96)$ & 0.69 & 7.65 & $\sim 6.2$ & 178 \\
\hline HD $76700 \mathrm{~b}$ & 085355.5153 & -664803.571 & G6V & 59.7 & $0.049(0.82)$ & 0.197 & 8.13 & $\sim 6.5$ & 207 \\
\hline * $51 \mathrm{Peg} \mathrm{b}$ & 225727.9805 & +204607.796 & G2.5IV & 15.4 & $0.0512(3.33)$ & 0.46 & 5.49 & $\sim 4.0$ & 51 \\
\hline$* v$ And $\mathrm{b}$ & 013647.8428 & +412419.652 & F8V & 13.5 & $0.059(4.37)$ & 0.69 & 4.09 & $\sim 2.7$ & 39 \\
\hline HD $49674 \mathrm{~b}$ & 65130.5164 & +405203.923 & G0 & 40.7 & $0.057(1.40)$ & 0.12 & 8.10 & $\sim 6.7$ & 122 \\
\hline HD $68988 \mathrm{~b}$ & 081822.1731 & +612738.599 & G0 & 58.9 & $0.071(1.21)$ & 1.90 & 8.21 & $\sim 6.8$ & 141 \\
\hline HD $168746 b$ & 182149.7832 & -115521.66 & G5 & 43.1 & $0.065(1.51)$ & 0.23 & 7.95 & $\sim 6.4$ & 113 \\
\hline$*$ HD $217107 \mathrm{~b}$ & 225815.5413 & -022343.386 & G8IV & 19.7 & $0.07(3.55)$ & 1.28 & 6.18 & $\sim 4.4$ & 48 \\
\hline HD $162020 \mathrm{~b}$ & 75038.3575 & -401906.056 & $\mathrm{~K} 2 \mathrm{~V}$ & 31.3 & $0.088(2.81)$ & 1.08 & 9.18 & $\sim 7.0$ & 61 \\
\hline HD $108147 \mathrm{~b}$ & 122546.2686 & -640119.516 & F8V & 38.6 & $0.104(2.69)$ & 0.41 & 7.00 & $\sim 5.7$ & 63 \\
\hline
\end{tabular}

(a) Minimum baseline needed to resolve planet and star pair at H-band (1.65 $\mu \mathrm{m}): \Delta \Theta=\frac{\lambda}{2 B}$. 